J Interv Card Electrophysiol. 2020 March ; 57(2): 233-240. doi:10.1007/s10840-019-00560-9.

\title{
Characterization of atrial flutter after pulmonary vein isolation by cryoballoon ablation
}

\author{
Jayson R. Baman ${ }^{1}$, Rachel M. Kaplan ${ }^{1}$, Celso L. Diaz ${ }^{1}$, Graham Peigh ${ }^{1}$, Aakash A. Bavishi ${ }^{1}$, \\ Amar Trivedi ${ }^{1}$, Jeremiah Wasserlauf ${ }^{1}$, Alexandru B. Chicos ${ }^{1}$, Rishi Arora, Susan Kim ${ }^{1}$, \\ Albert Lin ${ }^{1}$, Nishant Verma ${ }^{1}$, Bradley P. Knight ${ }^{1}$, Rod S. Passman ${ }^{1}$ \\ ${ }^{1}$ Division of Cardiology, Department of Medicine, and the Bluhm Cardiovascular Institute, \\ Northwestern Memorial Hospital, Northwestern University, 676 N St Claire Street; Suite 600, \\ Chicago, IL 60611, USA
}

\section{Abstract \\ Purpose-Pulmonary vein isolation (PVI) by cryoballoon ablation (CBA) has emerged as a commonly used technique for the treatment of atrial fibrillation. We sought to explore the incidence, risk factors for, and characterization of post-CBA-PVI atrial flutter.}

\begin{abstract}
Methods-We analyzed a prospective registry of patients who underwent CBA-PVI at a single institution. We included patients with more than 3 months of follow-up data and excluded those with a history of cavotricuspid isthmus (CTI) ablation. Locations of post-CBA-PVI atrial flutters were determined by analysis of intracardiac electrograms and electroanatomic maps.
\end{abstract}

Results-There were 556 patients included in the analysis. The mean age was $61.0 \pm 10.6$ years, $67.4 \%$ were male, the number of failed anti-arrhythmic medication trials was $1.2 \pm 0.8$, and the duration of atrial fibrillation pre-CBA was $54.3 \pm 69.1$ months. The 28 -mm second-generation cryoballoon was used almost exclusively. Over a median follow-up time of $22.7 \pm 17.9$ months, 25 $(4.5 \%)$ patients developed post-CBA-PVI atrial flutter after the 3-month blanking period. Of those 25 patients, $15(60 \%)$ underwent subsequent ablation to eliminate the atrial flutter circuit, with $60 \%$ being CTI-dependent and the remainder left-sided ( $p$ value not significant). Risk factors for the development of atrial flutter included NYHA class $\geq 2$ (OR 5.02, $p<0.001$ ), presence of baseline bundle branch block (OR 4.33, $p=0.006$ ), and left ventricular ejection fraction $<50 \%$ (OR 3.36, $p=0.007$ ).

Conclusions-The rate of post-CBA-PVI atrial flutter is low after the blanking period even with medium-term follow-up. The origin of atrial flutter is equally divided between the right and left atria.

\footnotetext{
${ }^{凶}$ Rod S. Passman, r-passman@ northwestern.edu.

Author contributions JRB Concept/design, data analysis/interpretation, drafting article.

RK Concept/design, critical revision of article.

CLD, GP, AAB Data collection, critical revision of article.

AT, JW, ABC, RA, SK, AL, NV Data acquisition, approval of article.

BPK Concept/design, critical revision of article.

RSP Concept/design, data analysis/interpretation, drafting article, critical revision of article.

The institutional review board at Northwestern University approved the study protocol.
} 


\section{Keywords}

Atrial fibrillation; Atrial flutter; Pulmonary vein isolation; Cryoballoon; Ablation

\section{Introduction}

The discovery that pulmonary veins play a critical role in the initiation and perpetuation of ectopic atrial discharge was a key finding that has informed the use of catheter-directed therapy for pulmonary vein isolation (PVI) for the management of AF [1]. At present, only radiofrequency energy, laser balloon, and cryothermy are approved energy sources for PVI. Atrial flutter is a known occurrence following PVI, though data suggest that the incidence may be lower after cryoballoon ablation (CBA) than radiofrequency ablation (RFA). The Fire and Ice Trial found that the combined rate of atrial flutter or atrial tachycardia was approximately three times lower after CBA-PVI than radiofrequency ablation for PVI (RFAPVI), although this finding did not achieve statistical significance [2]. Additional studies too have suggested a trend toward lower rates of post-ablation atrial tachyarrhythmias in the CBA-PVI population [3].

For years, the technique of choice for catheter-based PVI was RFA, but this technique has several limitations, including substantial operator dependence. The emergence of CBA, which is associated with shorter procedure times, reduced risk of cardiac perforation, and noninferior outcomes with regard to recurrence of atrial arrhythmia beyond the 3-month blanking period, offers a viable alternative $[2,4,5]$.

Since atrial flutter can occur following PVI, it is important to understand the pattern of postCBA-PVI atrial flutter [6]. There are limited data describing the incidence and intracardiac origin of atrial flutters following CBA-PVI [7, 8]. One smaller study of routine rhythm monitoring after first-time CBA-PVI found the post-CBA-PVI atrial flutter incidence as confirmed by EPS is 5.6\% (4 of 181 patients) with mean follow-up 16.6 months. This particular study did not identify any patients with left atrial macro-reentrant circuit [9].

In the enclosed report, we aim to investigate the incidence of and risk factors for post-CBAPVI atrial flutter and their anatomic location. Although previous studies have looked at the rates of recurrence of atrial tachyarrhythmias-including atrial fibrillation, atrial flutter, and atrial tachycardia—seldom have previous studies systematically examined the rates of atrial flutter, specifically, and characterized clinical risk factors and anatomic locations among the CBA-PVI population over long-term follow-up [3, 10, 11].

\section{Methods}

\subsection{Study population}

We performed a single-center retrospective cohort study of a prospectively maintained database of patients who underwent CBA-PVI at Northwestern Memorial Hospital, Chicago, IL, between July 2011 and December 2016. In total, we identified 674 consecutive cases but excluded 118 patients due to having $<3$ months of follow-up data $(n=63)$ or a history of prior cavotricuspid isthmus (CTI) ablation for typical atrial flutter $(n=55)$. The remaining 
556 patients comprised the study population. The institutional review board at Northwestern University approved the study protocol.

\subsection{Ablation procedure}

The index CBA-PVI procedures were performed by seven board-certified electrophysiologists with extensive ablation experience. Patients received conscious sedation or general anesthesia at the discretion of the treating physician. After obtaining access, transseptal puncture across the interatrial septum was performed using an SL1 or Preface sheath and Bayliss RF needle. Intravenous heparin was given with an ACT goal of $>300 \mathrm{~s}$. The Arctic Front cryoballoon catheter (Medtronic, Inc., Minneapolis, MN), sheath, and Achieve lasso catheter (Medtronic, Inc.) were introduced into the left atrium using the Cryosheath. 3D mapping was used at the discretion of the operator. A 28-mm secondgeneration cryoballoon was used in nearly all (> 95\%) cases. CBA was performed at the ostia of each pulmonary vein with an endpoint of entrance block, with pulmonary venogram obtained prior to each ablation discharge in order to confirm appropriate location and balloon occlusion of the ostia. Lesion duration evolved over time from two 4-min freezes per vein to two 3-min freezes per vein, with some operators limiting cryoablation to a single 3min application per vein when the time to effect was less than $30 \mathrm{~s}$. Target temperatures were -30 to $-55{ }^{\circ} \mathrm{C}$ for all patients, and esophageal monitoring was used for those patients receiving general anesthesia. We performed pacing to assess for exit block. Isoproterenol was not routinely given, nor were attempts to induce atrial flutter routinely made.

\subsection{Outcome measures}

Patients were followed at routine outpatient appointments at 2-3 months post-ablation and then every 6 months for a minimum of 2 years. Monitoring consisted of 3-week continuous monitoring off antiarrhythmic drugs at approximately 3 months, then $24-48 \mathrm{~h}$ of monitoring at 6-month intervals. Electrocardiograms (ECGs) were performed at every office visit and additional monitoring was performed in response to reported symptoms. ECGs were interpreted by three board-certified electrophysiologists. The identification of atrial flutter by Holter/event monitor was confirmed by a board-certified electrophysiologist. Smartphonebased rhythm monitoring or downloads from implanted devices were also used when available. In our statistical analyses, we specifically chose to exclude atrial flutters identified within the 3-month blanking period in compliance with the latest recommendations [12].

\subsection{Statistical analysis}

Continuous variables were compared by two-sided $t$ test of unknown variance and categorical variables were analyzed by chi-squared testing. For continuous variables, MannWhitney testing was also performed to account for non-normally distributed data. Statistical significance was defined as $p<0.05$. We additionally performed univariate binary studies, assessing odds ratios for the identification of risk factors associated with the primary endpoint. 


\subsection{Identification and classification of atrial flutter circuit location}

In patients who underwent EPS, location of the flutter was determined at the time of EPS and ablation by standard techniques including 3D mapping and entrainment analysis [13, 14]. We did not attempt to determine the localization of atrial flutter from surface ECG's because previous reports $[15,16]$ have shown this to be unreliable, especially in the postablation atria [17]. In Table 2, cases where atrial flutter was identified by ECG or Holter/ event monitor alone are specified as "Identified by surface tracing, unable to localize."

\section{Results}

We evaluated 556 of a total 674 consecutive patients who underwent CBA-PVI from 2011 to 2016. A total of 118 patients were excluded due to $<3$ months of follow-up or a history of prior cavotricuspid isthmus (CTI) ablation for typical atrial flutter. No patient received CTI ablation at the time of index CBA-PVI. The baseline characteristics of the study population are described in Table 1. The average age was $61.0 \pm 10.6$ years, $67.8 \%$ were male, $44.1 \%$ had a history of hypertension, $15.5 \%$ had ischemic cardiomyopathy, and mean LVEF was $56.1 \pm 9.8 \%$. The mean number of failed anti-arrhythmic drugs was $1.2 \pm 0.8$, and the duration of known atrial fibrillation was $54.3 \pm 69.1$ months. The numbers of patients with paroxysmal, persistent, and long-standing persistent atrial fibrillation were 293 (52.7\%), 79 (14.2\%), and 184 (33.1\%), respectively. Patients were followed for a median of $22.7 \pm 17.9$ months. Over this period, 221 total patients (39.7\%) developed recurrence of atrial fibrillation after the 3-month blanking period. The rates of atrial fibrillation recurrence after the blanking period were $34.1 \%(n=100), 43.0 \%(n=34)$, and $47.3 \%(n=87)$ based on paroxysmal, persistent, and long-standing persistent atrial fibrillation status, respectively.

Subjects who developed atrial flutter after the blanking period demonstrated higher rates of baseline bundle branch block (20.0\% versus $5.5 \%, p<0.01)$, more advanced NYHA functional class $(1.4 \pm 0.6$ versus $1.1 \pm 0.4, p=0.03)$, and larger left atrial diameter $(42.1 \pm$ $6.4 \mathrm{~mm}$ versus $39.0 \pm 6.7 \mathrm{~mm}, p=0.03)$.

\subsection{Incidence of atrial flutter}

Forty-three cases of post-CBA-PVI atrial flutter were identified, representing an overall rate of $7.7 \%$ across the study period. As shown in Fig. 1, the highest incidence of identified cases of atrial flutter occurred in the 3-month blanking period. Of the 18 patients who developed atrial flutter during the blanking period, 7 patients were noted to have atrial flutter during subsequent follow-up evaluations; 5 patients underwent cardioversion to extinguish atrial flutter during the blanking period, and there was no recurrence of future atrial flutter in these patients. Only 25 patients (4.5\%) developed atrial flutter beyond the blanking period, with 12 cases occurring within the first year of follow-up.

\subsection{Location of atrial flutter}

The locations of post-CBA-PVI atrial flutter in our study population are displayed in Table 2 and Figs. 1 and 2. Of the 25 patients with post-CBA-PVI atrial flutter beyond the blanking period, 15 (60\%) subjects underwent subsequent EPS. EPS found that these cases were approximately evenly distributed between CTI-dependent and left atrial locations. 
Reconnection of at least one pulmonary vein was present in 4 of the $12(33.3 \%)$ cases in which pulmonary vein interrogation was pursued. Of the 10 patients who did not undergo repeat EPS, 5 were treated with cardioversion alone, 1 was treated with anti-arrhythmic medication, and the remaining subjects either converted to sinus rhythm spontaneously ( $n=$ $2)$ or were lost to follow-up $(n=2)$.

\subsection{Identification of risk factors}

Table 3 demonstrates the findings from univariate analyses of odds ratios for suspected risk factors for the development of atrial flutter after the blanking period. Only patients who developed new atrial flutter after the blanking period were included in these analyses. Symptomatic heart failure (NYHA class 22 ), systolic dysfunction (LVEF $<50 \%$ ), and presence of bundle branch block and recurrence of atrial fibrillation more than 3 months after the index CBA-PVI were all associated with a significantly higher incidence of atrial flutter.

\section{Discussion}

In our study of 556 patients with median follow-up period of greater than 2 years, we demonstrated that the emergence of post-CBA-PVI atrial flutter beyond the blanking period is uncommon, with atrial flutters emerging at a similar proportion in the right and left atria. We additionally identified specific risk factors for the development of atrial flutter. These findings carry important implications regarding the clinical care of post-CBA-PVI patients.

In the absence of iatrogenic scar formation, such as after cardiac surgery or percutaneous ablation, most naturally occurring atrial flutter circuits are located in the right atrium. These "typical" atrial flutters propagate along various anatomic landmarks, including the crista terminalis and cavotricuspid isthmus (CTI). Previous studies have shown that the rates of post-RFA-PVI and post-CBA-PVI typical atrial flutter are statistically similar but trend toward decreased rates in the post-CBA-PVI population, which may be explained by effects from the different methods of energy delivery and resultant lesions of scar tissue. Our findings mirror a prior report that estimates the incidence of post-CBA-PVI atrial flutter to be approximately 5\% [9]. In a different smaller cohort study that analyzed typical atrial flutter after CBA-PVI, Akerstrom et al. reported rates of 3.3\% - similar to our findings [3]. This study also employed the use of second-generation $28-\mathrm{mm}$ cryoballoon in nearly all cases. Our study included significantly more CBA-PVI patients (556 versus 215) and patients at higher risk, including more advanced age (mean age 61.0 versus 57.7 years), higher BMI (29.5 versus 26.2), and higher rates of ischemic cardiomyopathy (15.5\% versus $5.6 \%)$. This study therefore builds on prior work by reproducing similar results in a higherrisk and larger population, while additionally identifying specific risk factors for the occurrence of post-ablation atrial flutter.

The findings of this study may also inform operators in planning of the ablation procedure. The PREVENT-AF Study I demonstrated that, in patients with atrial flutter and without atrial fibrillation, prophylactic pulmonary vein isolation at the time of CTI ablation successfully reduces the rates of future atrial fibrillation [18]. Some investigators have advocated for prophylactic CTI ablation at the time of PVI. While the most recent expert 
consensus statement provides a class I recommendation for adjunctive CTI ablation in patients with a history of typical atrial flutter or inducible CTI-dependent atrial flutter at the time of PVI [12], relatively little is known about the role of adjunctive CTI ablation in patients without that history. A propensity-matched analysis of $>800$ patients without inducible or documented atrial flutter found that prophylactic CTI at the time of first-time PVI procedure does not reduce long-term recurrence rates for AF, but further validation was certainly required and our study of a prospective cohort clarifies this conclusion [19].

In our study, the primary outcome of interest—post-CBA-PVI atrial flutter-was assessed in patients who met the prespecified inclusion criteria. Patients with new atrial flutter during the 3-month blanking period were excluded from the statistical analyses, in accordance with contemporary recommendations [12]. Additionally, we did not include presumptions of atrial flutter location in patients who did not undergo subsequent EPS. Numerous studies have shown that atrial flutter localization, especially in the post-ablation atria, based on surface tracing is unreliable [15-17].

We found that at least one pulmonary vein was reconnected in $33 \%$ of patients. This is similar to previous reports, where the incidence of reconnection is reported to be $>50 \%$ [20]; however, it should be noted that not all patients underwent assessment for pulmonary vein reconnection in our series. A meta-analysis of nearly 700 patients found that the risk of recurrent atrial fibrillation is significantly associated with pulmonary vein reconnection; however, data investigating the relationship between new atrial flutter and pulmonary vein reconnection are scarce [20].

We identified left atrial diameter, NYHA class, left ventricular ejection fraction, bundle branch block, and late recurrence of atrial fibrillation as risk factors for the development of atrial flutter after CBA-PVI. The causal relationships between risk factors for and the development of atrial flutter have been an active area of study [21]. Larger left and right atrial diameters have previously been linked to increased burden of atrial flutter [22]. In our study, we did not collect data on right atrial diameter, but it is possible that left atrial dilatation could be a marker for biatrial dilatation, which can serve as a geometric substrate for atrial flutter formation. Local inflammation from atrial stretching may induce areas of micro-scar formation that may predispose patients to developing atrial flutter. Additionally, atrial flutter can induce atrial remodeling in itself [23, 24]. It is possible that these patients may have had preexisting substrate for atrial flutter prior to PVI, such that the obliteration of the dominant AF rhythm unmasked an underlying, macro-reentrant atrial flutter circuit.

Advanced NYHA class and reduced LVEF may be surrogates for underlying myocardial dysfunction at a cellular level. The activation of fibroblasts in heart failure has been linked to biomechanical changes that can occur outside of the local area of injury. In such a way, myocardial inflammation can predispose individuals for subsequent arrhyth-mogenic phenomena, including atrial flutter [25]. Atrial fibrillation could already be such a consequence in these patients; thus, it would not be too surprising that atrial flutters emerge in these same high-risk patients [26]. Similarly, the presence of bundle branch block indicates disruption of the His-Purkinje conducting system, which is oftentimes due to fibrotic or ischemic insult. These same pathophysiologic processes are predisposing 
conditions for atrial flutter. In this way, the identification of bundle branch block in this particular set of patients may be an early marker to risk-stratify patients who could develop atrial flutter. This supposes an associative, rather than a causal, relationship.

These risk factors should be considered following CBA-PVI, as the emergence of atrial flutter has important implications for anticoagulation and potential subsequent ablations.

Importantly, we showed that only $4.5 \%$ of patients who underwent CBA-PVI would develop future atrial flutter after the blanking period, with $1.6 \%$ of the study population developing EPS-confirmed CTI-dependent atrial flutter. Thus, the prophylactic addition of CTI ablation to CBA-PVI is unlikely to have significant impact on long-term recurrence rates and should only be considered in "high-risk" individuals if at all.

\subsection{Limitations}

This is a retrospective single-center study. Although the study population represents a heterogeneous group, there may be unavoidable bias related to the fact that these patients were treated at a single high-volume referral center. Second, some patients who went on to develop atrial flutter did not undergo a subsequent EPS, thus the ability to localize the atrial flutter circuit in these patients was limited. Interpretation of surface electrocardiograms in these patients is compromised by their history of previous left atrial ablation. We recognize that other arrhythmias (atrial tachycardia, organized atrial fibrillation) can appear similarly to atrial flutter on surface electrocardiogram. In this way, our estimates of atrial flutter rate by including those recognized by ECG and Holter/event monitor alone could represent an overestimation of the true value. Conversely, since paroxysmal atrial flutter may have occurred outside of periods of ambulatory cardiac rhythm monitoring, the incidence of atrial flutter reported above could actually be an underestimate. Although these limitations exist, we are confident that our protocol for routine post-CBA-PVI monitoring provides us with valuable information to estimate our outcome of interest.

\section{Conclusions}

We report the incidence of post-CBA-PVI atrial flutter in a large registry with $>2$ years of follow-up and without history of previous CTI ablation. The overall incidence of post-CBAPVI atrial flutter after the blanking period is $4.5 \%$, with only $1.6 \%$ of all patients developing confirmed CTI-dependent atrial flutter. The plurality of atrial flutter cases occurs during the blanking period. Additionally, we identified multiple factors-echocardiographic and clinical - that predicted patients at increased risk of post-CBA-PVI atrial flutter.

\section{Acknowledgments}

Conflict of interest Bradley P. Knight receives honoraria for consulting and speaking for Medtronic Inc. Rod S. Passman receives research support, consulting fees, and speaker fees from Medtronic; royalties from UpToDate; and research support from Pfizer and Kardia. Northwestern University receives fellowship support from Medtronic, Inc. 


\section{References}

1. Haissaguerre M, Jais P, Shah DC, Takahashi A, Hocini M, Quiniou G, et al. Spontaneous initiation of atrial fibrillation by ectopic beats originating in the pulmonary veins. N Engl J Med. 1998;339(10): 659-66. [PubMed: 9725923]

2. Kuck KH, Brugada J, Furnkranz A, Metzner A, Ouyang F, Chun KR, et al. Cryoballoon or radiofrequency ablation for paroxysmal atrial fibrillation. N Engl J Med. 2016;374(23):2235-45. [PubMed: 27042964]

3. Akerstrom F, Bastani H, Insulander P, Schwieler J, Arias MA, Jensen-Urstad M. Comparison of regular atrial tachycardia incidence after circumferential radiofrequency versus cryoballoon pulmonary vein isolation in real-life practice. J Cardiovasc Electrophysiol. 2014;25(9):948-52. [PubMed: 24698206]

4. Wasserlauf J, Pelchovitz DJ, Rhyner J, Verma N, Bohn M, Li Z, et al. Cryoballoon versus radiofrequency catheter ablation for paroxysmal atrial fibrillation. Pacing Clin Electrophysiol : PACE. 2015;38(4):483-9. [PubMed: 25627795]

5. Straube F, Dorwarth U, Ammar-Busch S, Peter T, Noelker G, Massa T, et al. First-line catheter ablation of paroxysmal atrial fibrillation: outcome of radiofrequency vs. cryoballoon pulmonary vein isolation. Europace. 2016;18(3):368-75. [PubMed: 26504108]

6. Lee G, Sanders P, Kalman JM. Catheter ablation of atrial arrhythmias: state of the art. Lancet (London, England). 2012;380(9852): 1509-19.

7. Coutino HE, de Asmundis C, Mugnai G, Moran D, De Regibus V, Stroker E, et al. Repeat procedures after second-generation cryoballoon ablation as an index procedure for persistent atrial fibrillation: one-year follow-up. J Interv Card Electrophysiol. 2016;47(3):365-71. [PubMed: 27562140]

8. East C, Phan T, Filardo G, Franklin J, Donsky A, Wheelan KR, et al. Repeat ablation and hospitalization following cryoballoon ablation of atrial fibrillation at a single tertiary medical center. Proceedings. 2017;30(1):3-6.

9. Mikhaylov EN, Bhagwandien R, Janse PA, Theuns DA, Szili-Torok T. Regular atrial tachycardias developing after cryoballoon pulmonary vein isolation: incidence, characteristics, and predictors. Europace. 2013;15(12):1710-7. [PubMed: 23689485]

10. Cardoso R, Mendirichaga R, Fernandes G, Healy C, Lambrakos LK, Viles-Gonzalez JF, et al. Cryoballoon versus radiofrequency catheter ablation in atrial fibrillation: a meta-analysis. $\mathrm{J}$ Cardiovasc Electrophysiol. 2016;27(10):1151-9. [PubMed: 27422848]

11. Hermida A, Kubala M, Traulle S, Buiciuc O, Quenum S, Hermida JS. Prevalence and predictive factors of left atrial tachycardia occurring after second-generation cryoballoon ablation of atrial fibrillation. J Cardiovasc Electrophysiol. 2018;29(1):46-54. [PubMed: 29024212]

12. Calkins H, Hindricks G, Cappato R, Kim YH, Saad EB, Aguinaga L, et al. 2017 HRS/EHRA/ ECAS/APHRS/SOLAECE expert consensus statement on catheter and surgical ablation of atrial fibrillation. Heart Rhythm. 2017;14(10):e275-444. [PubMed: 28506916]

13. Deo R, Berger R. The clinical utility of entrainment pacing. J Cardiovasc Electrophysiol. 2009;20(4):466-70. [PubMed: 19207769]

14. Pedrinazzi C, Durin O, Mascioli G, Curnis A, Raddino R, Inama G, et al. Atrial flutter: from ECG to electroanatomical 3D mapping. Heart Int. 2006;2(3-4):161. [PubMed: 21977266]

15. Cosio FG. Atrial flutter, typical and atypical: a review. Arrhythmia Electrophysiol Rev. 2017;6(2):55-62.

16. Medi C, Kalman JM. Prediction of the atrial flutter circuit location from the surface electrocardiogram. Europace. 2008;10(7):786-96. [PubMed: 18456647]

17. Gerstenfeld EP, Dixit S, Bala R, Callans DJ, Lin D, Sauer W, et al. Surface electrocardiogram characteristics of atrial tachycardias occurring after pulmonary vein isolation. Heart Rhythm. 2007;4(9): 1136-43. [PubMed: 17765610]

18. Steinberg JS, Romanov A, Musat D, Preminger M, Bayramova S, Artyomenko S, et al. Prophylactic pulmonary vein isolation during isthmus ablation for atrial flutter: the PReVENT AF Study I. Heart Rhythm. 2014;11(9):1567-72. [PubMed: 24832767] 
19. Mesquita J, Ferreira AM, Cavaco D, Carmo P, Madeira M, Freitas P, et al. Impact of prophylactic cavotricuspid isthmus ablation in atrial fibrillation recurrence after a first pulmonary vein isolation procedure. Int J Cardiol. 2018;259:82-7. [PubMed: 29579616]

20. Nery PB, Belliveau D, Nair GM, Bernick J, Redpath CJ, Szczotka A, et al. Relationship between pulmonary vein reconnection and atrial fibrillation recurrence: A Systematic Review and MetaAnalysis. JACC Clin Electrophysiol. 2016;2(4):474-83. [PubMed: 29759868]

21. Rahman F, Wang N, Yin X, Ellinor PT, Lubitz SA, LeLorier PA, et al. Atrial flutter: clinical risk factors and adverse outcomes in the Framingham Heart Study. Heart Rhythm. 2016;13(1):233-40. [PubMed: 26226213]

22. Da Costa A, Mourot S, Romeyer-Bouchard C, Thevenin J, Samuel B, Kihel A, et al. Anatomic and electrophysiological differences between chronic and paroxysmal forms of common atrial flutter and comparison with controls. Pacing Clin Electrophysiol: PACE. 2004;27(9):1202-11. [PubMed: 15461709]

23. Medi C, Teh AW, Roberts-Thomson K, Morton JB, Kistler PM, Kalman JM. Right atrial remodeling is more advanced in patients with atrial flutter than with atrial fibrillation. J Cardiovasc Electrophysiol. 2012;23(10):1067-72. [PubMed: 22612914]

24. Nattel S, Shiroshita-Takeshita A, Cardin S, Pelletier P. Mechanisms of atrial remodeling and clinical relevance. Curr Opin Cardiol. 2005;20(1):21-5. [PubMed: 15596955]

25. Talman V, Ruskoaho H. Cardiac fibrosis in myocardial infarction-from repair and remodeling to regeneration. Cell Tissue Res. 2016;365(3):563-81. [PubMed: 27324127]

26. Manolis AS. Contemporary diagnosis and management of atrial flutter: a continuum of atrial fibrillation and vice versa? Cardiol Rev. 2017;25(6):289-97. [PubMed: 28832375] 


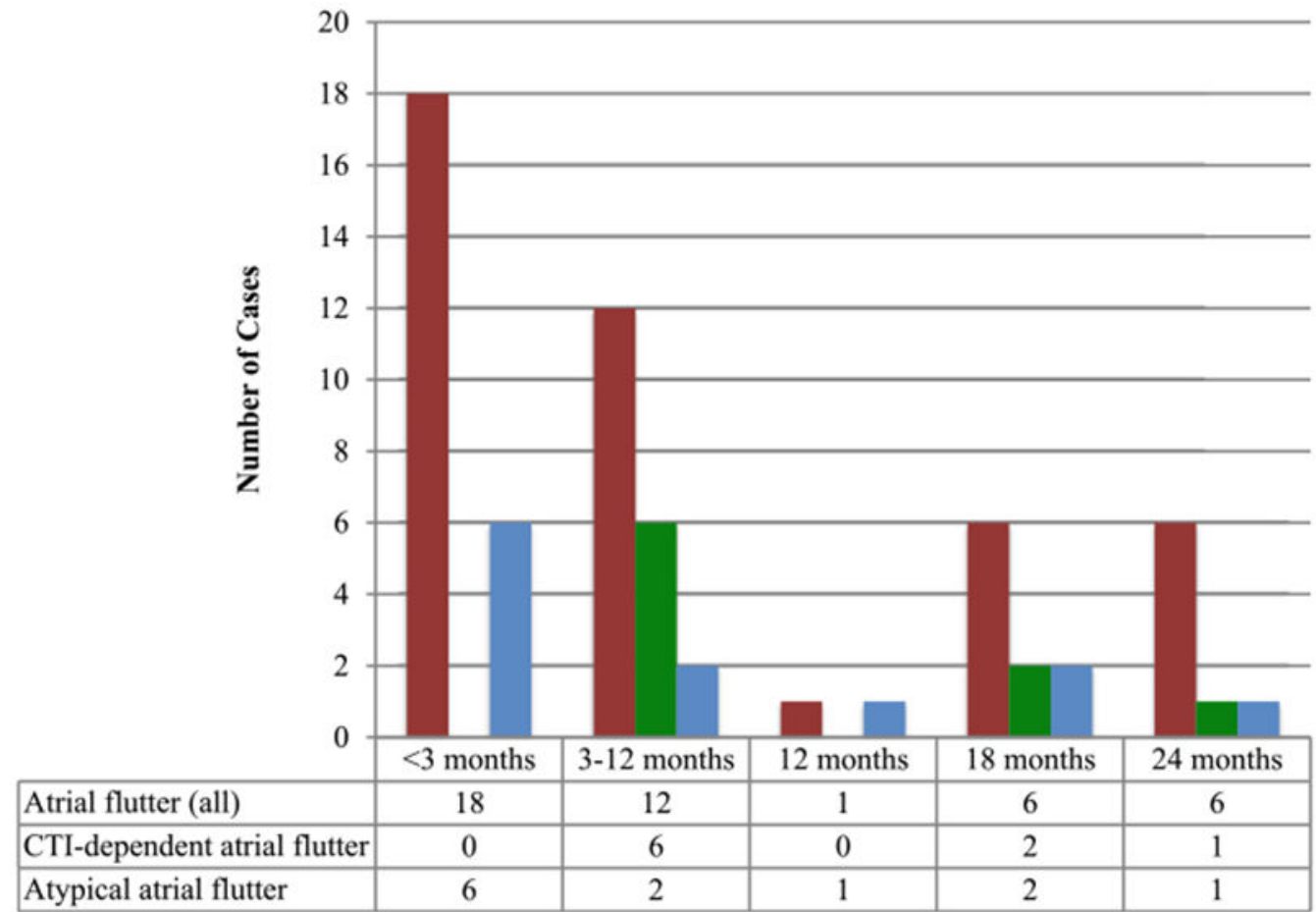

- Atrial flutter (all)

- CTI-dependent atrial flutter = Atypical atrial flutter

Fig. 1.

Number of atrial flutter cases identified, as indicated by time to recognition after CBA-PVI.

"Atrial flutters (all)" includes all atrial flutters that were identified by electrocardiogram, ambulatory monitor, and/or electrophysiology study (EPS). The locations listed are those confirmed by EPS 


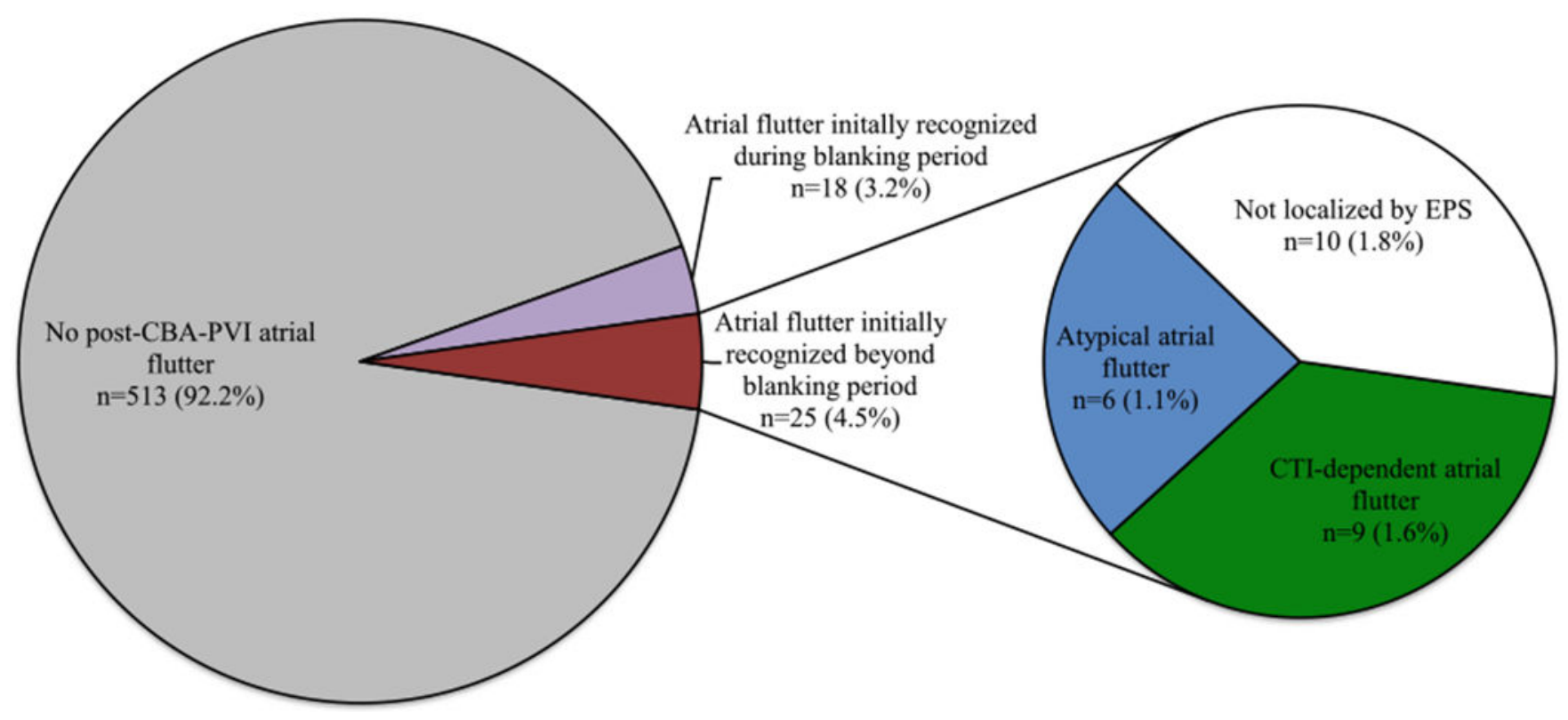

Fig. 2.

Graphical representation of the proportion of patients with a diagnosis of atrial flutter following CBA-PVI. CBA-PVI pulmonary vein isolation by cryoballoon ablation, EPS electrophysiology study 


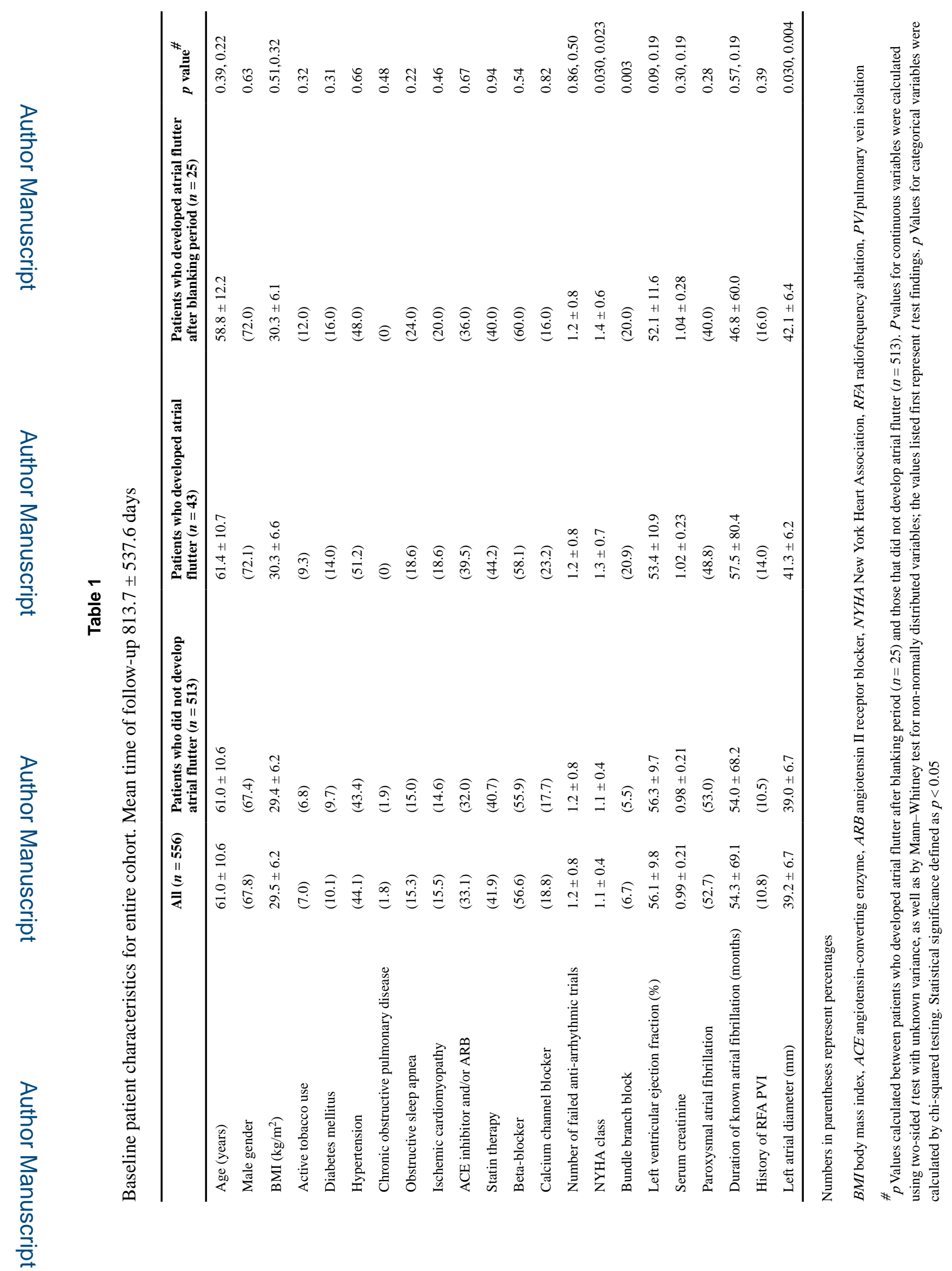

J Interv Card Electrophysiol. Author manuscript; available in PMC 2020 March 28. 


\section{롤 \\ 공}

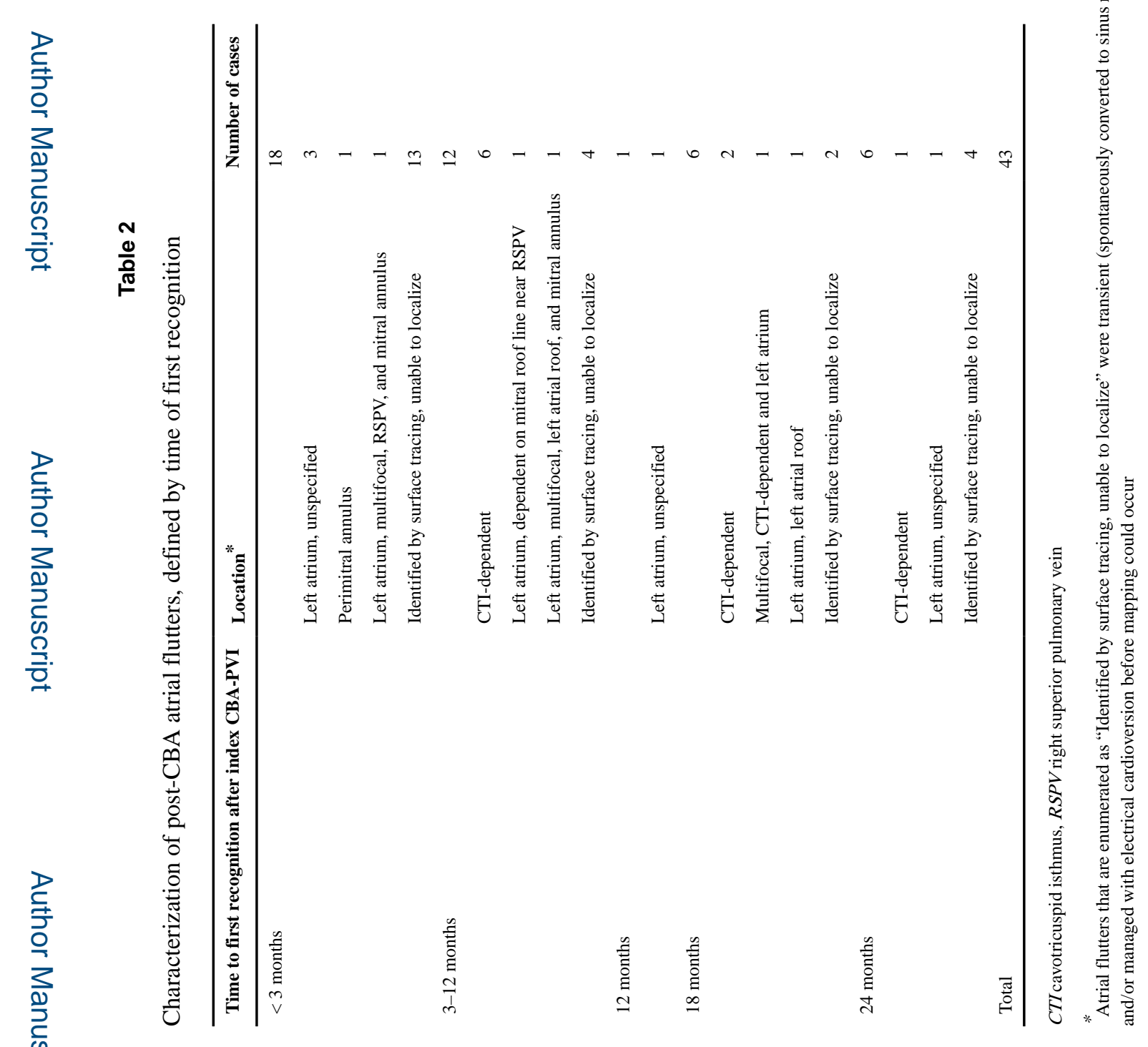




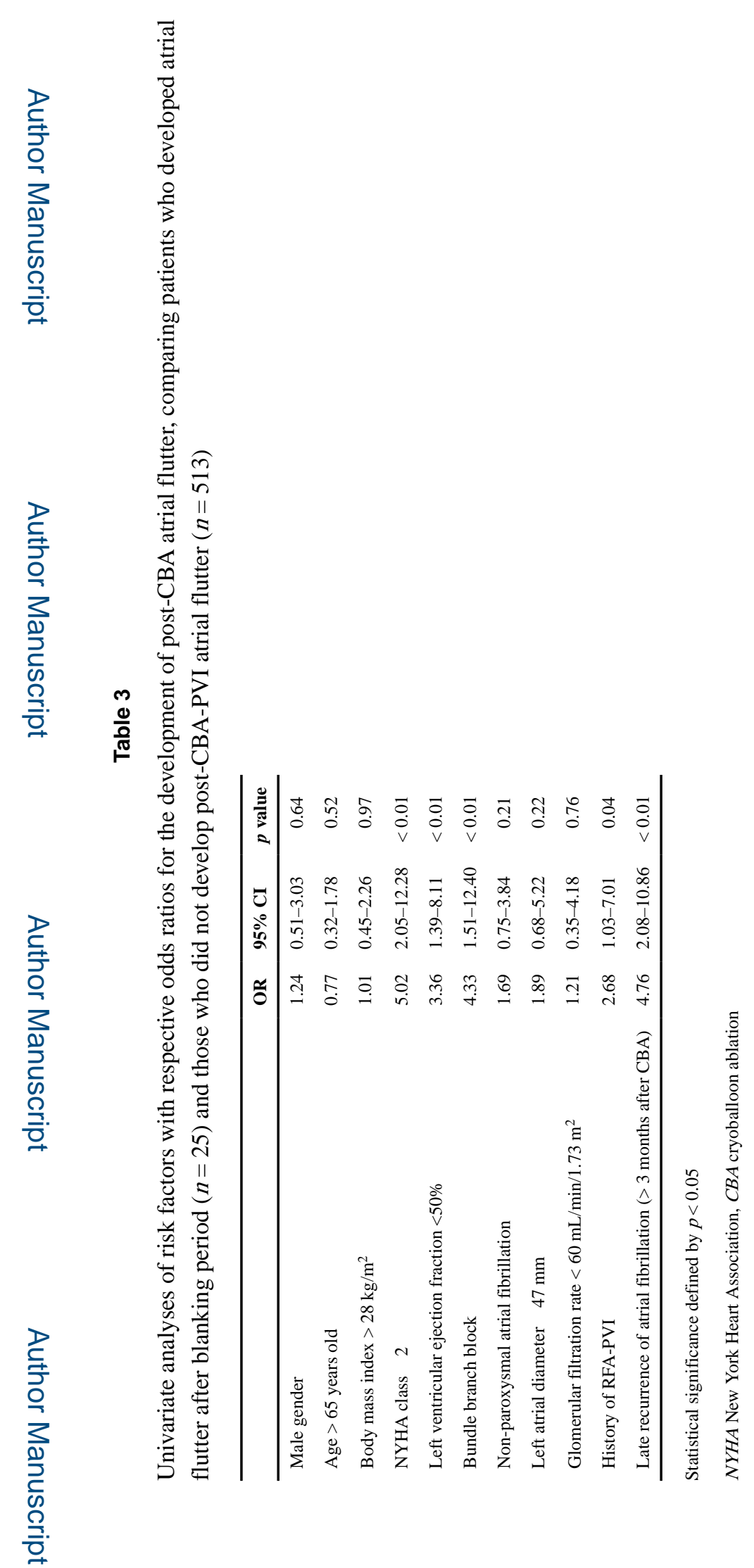

J Interv Card Electrophysiol. Author manuscript; available in PMC 2020 March 28. 